\title{
Sharp-line electroluminescence from individual quantum dots by resonant tunneling injection of carriers
}

\author{
L. Turyanska, A. Baumgartner, A. Chaggar, A. Patanè, ${ }^{\text {a) }}$ L. Eaves, and M. Henini \\ School of Physics and Astronomy, University of Nottingham, Nottingham NG7 2RD, United Kingdom
}

(Received 11 April 2006; accepted 29 June 2006; published online 29 August 2006)

\begin{abstract}
We report sharp electroluminescence lines from individual self-assembled InAs quantum dots (QDs) excited by resonant tunneling injection of carriers from the $n$ - and $p$-doped GaAs layers of a $p-i-n$ diode. Bias-tunable tunneling of carriers into the dots provides a means of controlling injection and light emission from a small number of individual dots within a large ensemble. We also show that the extent of carrier energy relaxation prior to recombination can be controlled by tailoring the morphology of the QD layer. (C) 2006 American Institute of Physics. [DOI: 10.1063/1.2337540]
\end{abstract}

Semiconductor nanocrystals, also known as quantum dots (QDs), are artificial nanostructures with discrete and narrow electronic energy levels, similar to those observed in atomic physics. ${ }^{1,2}$ Sharp-line optical emission spectra of QDs have been extensively studied by high-resolution spatially resolved optical techniques, such as near-field scanning optical microscopy and microphotoluminescence. Selective emission from a small number of dots within a large ensemble has also been achieved by incorporating the dots in lithographically patterned small area diodes, ${ }^{2-6}$ microcavity pillars, ${ }^{7}$ and nanowires. ${ }^{8,9}$ These studies have provided detailed information about the electronic properties of the dots and have also formed the basis for novel applications of QDs as single photon emitters for spintronics ${ }^{10}$ and in quantum information processing. ${ }^{11}$

In this work, we investigate a large ensemble of selfassembled InAs QDs incorporated in the intrinsic region $(i)$ of a large area GaAs $p-i-n$ diode. We demonstrate that biastunable tunneling of carriers into the dots provides us with a means of exciting a small number of QDs out of a very large ensemble $\left(10^{7}-10^{8}\right)$ without resorting to submicron scale lithography or spatially resolved optical techniques. We report sharp electroluminescence (EL) lines from individual QDs excited by resonant tunneling injection of carriers from the $n$ - and $p$-doped GaAs layers of the diode. We present a study of the dot EL under various applied biases and for different diode designs. This technique, EL excitation, complements the more familiar photoluminescence excitation spectroscopy as a probe of excitation and relaxation of carriers.

Our $p-i$ - $n$ diodes were grown by molecular beam epitaxy (MBE) and incorporate a layer of self-assembled InAs QDs in the intrinsic $(i)$ region, see Fig. 1(a). Sample A1 was grown on a $n^{+}$-GaAs substrate with the following order of growth layers: A $0.7 \mu \mathrm{m}$ thick $n^{+}$-doped GaAs buffer layer $\left(n^{+}: 4 \times 10^{18} \mathrm{~cm}^{-3}\right)$ followed by a $100 \mathrm{~nm}$ thick $n$-doped GaAs $\left(n: 4 \times 10^{16} \mathrm{~cm}^{-3}\right)$; an undoped, intrinsic region, which consists, respectively, of a $100 \mathrm{~nm}$ thick GaAs layer, an InAs layer of thickness $L=2 \mathrm{ML}$, and a $60 \mathrm{~nm}$ thick GaAs layer; finally, the structure is capped with a $500 \mathrm{~nm}$ thick $p^{+}$-doped GaAs top contact layer $\left(p^{+}: 2 \times 10^{18} \mathrm{~cm}^{-3}\right)$. The structure was grown at $600{ }^{\circ} \mathrm{C}$ except for the InAs layer and the over-

\footnotetext{
a) Author to whom correspondence should be addressed; electronic mail:
} amalia.patane@nottingham.ac.uk grown GaAs cap layer, which were grown at 450 and $500{ }^{\circ} \mathrm{C}$, respectively.

Samples B1 and B2 were grown on a semi-insulating GaAs substrate. They differ from sample A1 by having a double AlAs barrier resonant tunneling structure in the central intrinsic region. Their growth scheme is as follows: A $120 \mathrm{~nm}$ thick $p^{+}$-doped GaAs buffer layer $\left(p^{+}: 2\right.$ $\times 10^{18} \mathrm{~cm}^{-3}$ ) followed by a $30 \mathrm{~nm}$ thick $p$-doped GaAs $\left(p: 5 \times 10^{17} \mathrm{~cm}^{-3}\right)$; an undoped, intrinsic region $(i)$, which consists of a $2.9 \mathrm{~nm}$ thick AlAs layer, a $2.0 \mathrm{~nm}$ thick GaAs layer, an InAs layer of thickness $L=1.8 \mathrm{ML}$, a $10 \mathrm{~nm}$ thick GaAs layer, a $2.9 \mathrm{~nm}$ thick AlAs layer, and a $20 \mathrm{~nm}$ thick GaAs layer; the growth is completed by a $50 \mathrm{~nm}$ thick $n$-doped GaAs layer $\left(n: 2 \times 10^{16} \mathrm{~cm}^{-3}\right)$, an $80 \mathrm{~nm}$ thick $n$-doped GaAs layer $\left(n: 2 \times 10^{17} \mathrm{~cm}^{-3}\right)$, and a $100 \mathrm{~nm}$ thick

(a)
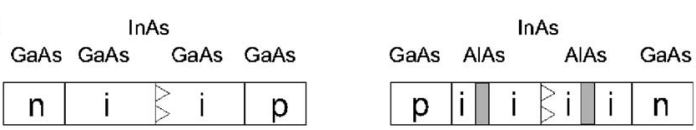

(b)
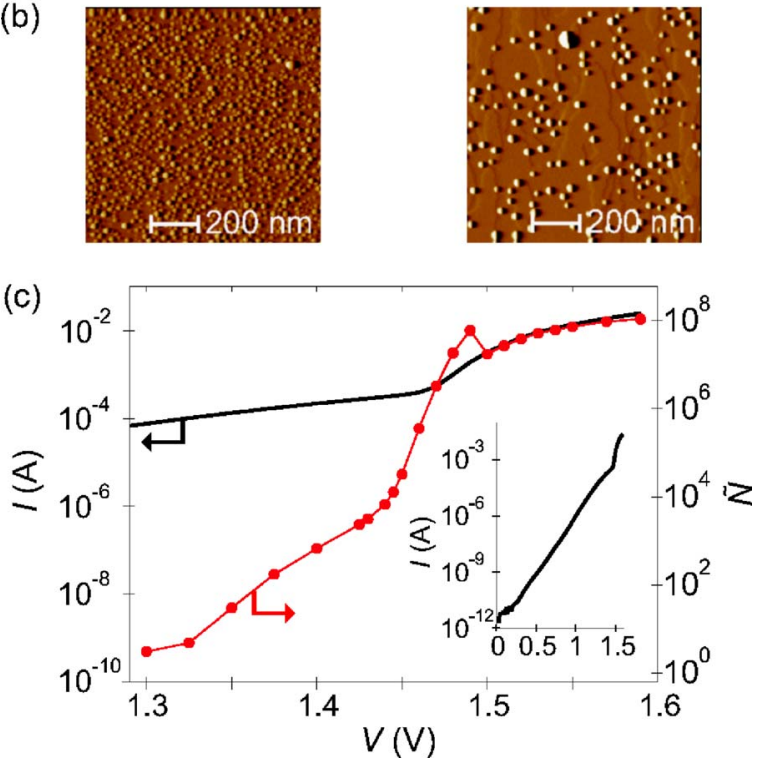

FIG. 1. (Color online) (a) Layout of $p-i-n$ diodes with design of types A (left) and B (right). (b) Atomic force microscopy images of high (left) and low (right) density self-assembled InAs QDs. (c) Left axis: currrent $I$ vs applied voltage $V$ at $T=10 \mathrm{~K}$ for sample A1. Right axis: bias dependence of the number of excited dots, $\widetilde{N}$. Inset: $I-V$ curve in the bias range of $0-$ $+1.5 \mathrm{~V}$. The mesa diameter is $400 \mu \mathrm{m}$. 
(a)
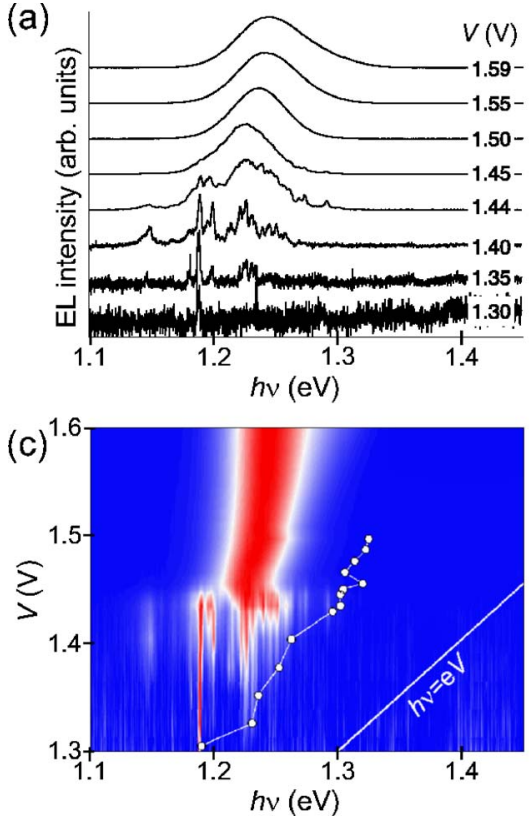

(b)

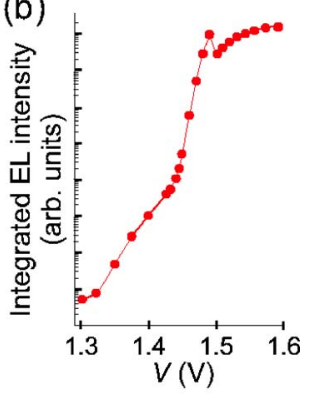

(d)

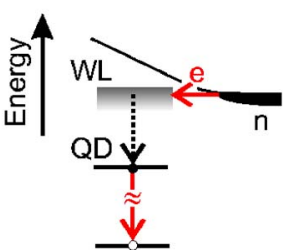

FIG. 2. (Color online) (a) Normalized EL spectra at different applied biases $V$ at $T=10 \mathrm{~K}$ for sample A1. The spectra are normalized to the peak intensity. (b) Dependence of the integrated intensity of the EL spectrum on applied bias. (c) Color plot of the normalized EL intensity vs photon energy $h v$ and applied bias $V$ for sample A1. The continuous line represents the condition $h v=e V$. The full dots represent the high-energy edge for the rise of EL signal. Red, white, and blue colors correspond to high, intermediate, and low EL intensities, respectively. The mesa diameter is $400 \mu \mathrm{m}$. (d) Schematic diagram of carrier tunneling, energy relaxation, and radiative recombination processes.

$n^{+}$-doped GaAs top layer $\left(n: 4 \times 10^{18} \mathrm{~cm}^{-3}\right)$. These structures were grown at $550{ }^{\circ} \mathrm{C}$ except for the InAs layer and the remaining overgrown layers which were grown at $500{ }^{\circ} \mathrm{C}$. In particular, in sample B1, the InAs layer was also annealed for $90 \mathrm{~s}$ at $540{ }^{\circ} \mathrm{C}$ before the GaAs overgrowth to facilitate the formation of large and low density dots. ${ }^{12}$ The dot morphology was studied by atomic force microscopy (AFM) on additional MBE wafers having a similar growth scheme but with the growth terminated after the InAs layer, see Fig. 1(b). In samples A1 and B2, the dots have a density of $\sim 10^{11} \mathrm{~cm}^{-2}$, a mean diameter of $\sim 20 \mathrm{~nm}$, and a mean height of $\sim 2 \mathrm{~nm}$. In sample B1, the dots have a lower density of $\sim 10^{10} \mathrm{~cm}^{-2}$ and larger mean diameter and height.

The $p-i-n$ structures were processed using conventional optical lithography into circular mesas of diameters $d=400$ and $200 \mu \mathrm{m}$. A ring-shaped electrical contact was fabricated on top of the mesa to permit optical access to the sample for measurements of EL and current-voltage $I-V$ characteristics. In all structures, the $I-V$ curves show diode-like behavior with a marked increase in the current for applied forward biases around $V=+1.5 \mathrm{~V}$, corresponding to the lowtemperature flatband condition of GaAs $p-i-n$ diodes, see inset of Fig. 1(c). Note, however, that a significant current flows at lower voltages down to and below $V \sim+1.3 \mathrm{~V}$. Similar behavior was reported in previous work and attributed to tunneling into the dots of carriers injected from the $n$ and $p$ layers of the diode. ${ }^{13}$

Figure 2(a) shows the low-temperature $(T=10 \mathrm{~K})$ normalized EL spectra of sample A1 for a range of applied biases. The bias dependence of the integrated intensity of the EL spectrum is shown in Fig. 2(b). At high bias, we observe an intense bell-shaped EL band from the QD ensemble. This Downloaded 30 Aug 2006 to 128.243.220.21. Redistribution subject evolves into a series of sharp lines with lower intensity as $V$ is decreased below the flatband condition. These sharp lines have a narrow energy width $(<1 \mathrm{meV})$ and their energy is independent of $V$. To track the evolution of the EL spectrum with varying $V$, in Fig. 2(c) we include a color-scale plot of the normalized EL intensity versus bias $V$ and photon energy $h v$. This shows clearly the redshift of the broad QD band as the voltage is decreased below $V \sim+1.6 \mathrm{~V}$ followed by the fragmentation of the spectrum into sharp lines for $V<V^{*}$ $\sim 1.44 \mathrm{~V}$. This characteristic voltage corresponds to the bias required for injection of carriers into the energy states of the InAs wetting layer, whose low-temperature absorption/ emission edge is typically observed at $h v \sim 1.4 \mathrm{eV}$.,14

In the regime of nonresonant excitation of the dots $(V$ $>V^{*}$ ), electrons and holes are injected uniformly across the mesa and all dots contribute to the EL spectrum, thus generating a broad and intense emission as observed in our experiment. In contrast, at lower bias, the applied voltage tunes selectively electron and hole injection into a small number of QDs within the large ensemble. The sharp EL lines persist at applied biases as low as $V=+1.3 \mathrm{~V}$, well below the flatband condition. Therefore, the electrons and holes which enter the QDs are tunneling through potential barriers, either directly from the $p$ - and $n$-doped contact layers or else through the low-energy localized states of the InAs wetting layer subband.

To interpret these data, we consider the integrated area $A$ of the QD EL spectrum. At a given bias, $A$ is equal to the radiative component $I_{r}$ of the electrical current $I$. The radiative component is given by $I_{r}(V)=\tilde{N} e \tau^{-1}$, where $\tau=10^{-9} \mathrm{~s}$ is the radiative lifetime of an exciton in the $\operatorname{dot}^{15} \tilde{N}$ $=\sum_{j=1}^{N} f_{j}(V)$ is the number of excited quantum dots, $f_{j}(V)$ defines the occupancy with an electron-hole pair of a particular $\operatorname{dot}(j)$ within the ensemble, and $N$ is the total number of dots in our mesa. By assuming that the quantum efficiency is close to unity at high bias, i.e., $I=I_{r}=A$ for $V>+1.5 \mathrm{~V}$, we can calibrate $I_{r}(V)$ and determine the value of $\tilde{N}=A \tau / e$ at all bias voltages. Our data and analysis indicate that for $V>+1.5 \mathrm{~V}, \widetilde{N}$ corresponds closely to the total number of dots $\left(N=10^{8}\right)$ in our large diameter mesa $(d=400 \mu \mathrm{m})$ as estimated from our AFM images, i.e., all dots are emitting at a rate $\tau^{-1}$. Below the flatband condition, $\tilde{N}$ falls rapidly and we deduce $\widetilde{N} \cong 3$ at $V \sim+1.3 \mathrm{~V}$, see Fig. 1(c). Around this bias, we observe only a few emission lines in the EL spectrum. We therefore conclude that these are due to emission from a very small number of individual dots, each with $f_{j}(V) \sim 1$.

We observe a similar fragmentation of the QD EL spectrum in all diodes of types A and B and in diodes incorporating dots with different dot sizes and densities. However, comparing experiments on various structures, we found that samples with high $\left(10^{11} \mathrm{~cm}^{-2}\right.$; sample A1 and sample B2) and low density $\left(10^{10} \mathrm{~cm}^{-2}\right.$; sample B1) dots have different behaviors. For low density dots, we find marked differences in the bias dependence of the QD emission, see EL spectra of sample B1 in Fig. 2(a). Of particular interest is the energy difference $\Delta \mathrm{E}$ between the excitation energy $e V$ provided by the applied bias and the high-energy edge $h v_{t}$ of the EL emission [see open circles and continuous line in the color plot of Figs. 2(c) and 3(c)]. In sample A1, with high QD density, $\Delta \mathrm{E}$ remains large over an extended range of bias: to AlP license or copyright, see http://apl.aip.org/apl/copyright.jsp 
(a)
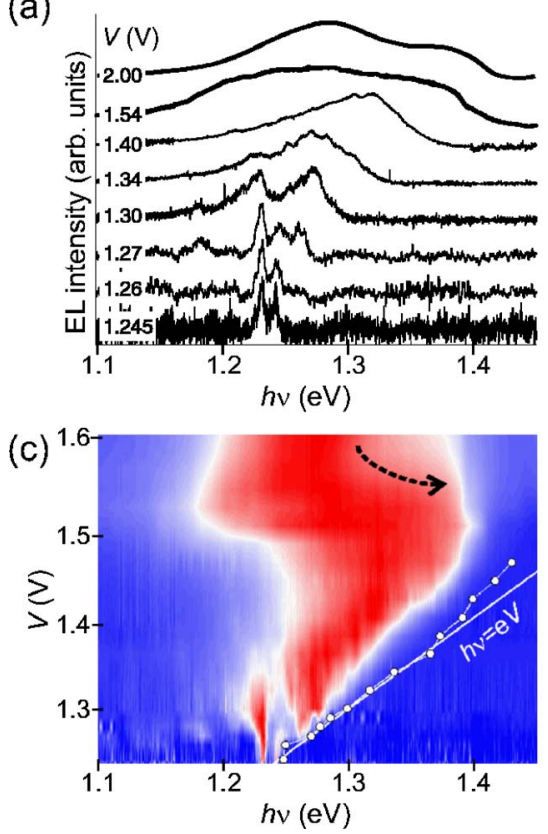

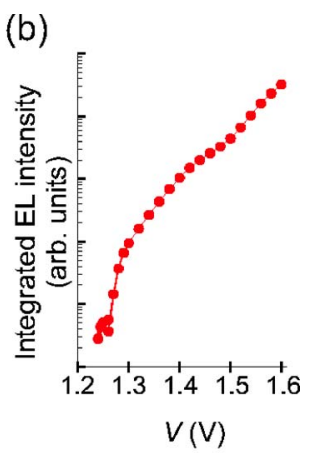

(d)

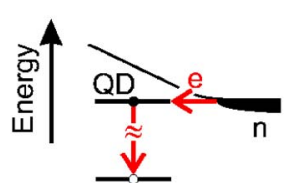

FIG. 3. (Color online) (a) Normalized EL spectra at different applied biases $V$ at $T=10 \mathrm{~K}$ for sample B1. The spectra are normalized to the peak intensity. (b) Dependence of the integrated intensity of the EL spectrum on applied bias. (b) Color plot of the normalized EL intensity vs photon energy $h v$ and applied bias $V$ for sample B1. The continuous line represents the condition $h v=e V$. The full dots represent the high-energy onset for the rise of EL signal. Red, white, and blue colors correspond to high, intermediate, and low EL intensities, respectively. The arrow indicates the blueshift of the EL emission as the bias is decreased below flatband condition. The mesa diameter is $200 \mu \mathrm{m}$. (d) Schematic diagram of carrier tunneling and recombination processes.

$\Delta \mathrm{E}=e V-h v_{t}=150 \mathrm{meV}$ at $V=+1.45 \mathrm{~V}$ and reduces to $\Delta \mathrm{E}$ $\sim 100 \mathrm{meV}$ at $V=+1.3 \mathrm{~V}$. This indicates that the electrically injected electron-hole pairs relax a considerable amount of energy $(\sim 100 \mathrm{meV})$ prior to radiative recombination from the ground states of the QDs; see Fig. 2(d). In contrast, in device B1 with low QD density $\left(\sim 10^{10} \mathrm{~cm}^{-2}\right)$, we observe only a small energy loss: $\Delta \mathrm{E} \sim 30 \mathrm{meV}$ at $V=1.45 \mathrm{~V}$ and $\Delta \mathrm{E}<1 \mathrm{meV}$ at $V=+1.3 \mathrm{~V}$. This small value of $\Delta \mathrm{E}$ at $1.3 \mathrm{~V}$ means that the electron and hole energies of the emitting $\mathrm{QD}(\mathrm{s})$ are resonantly aligned within the thermal energy $\left(k_{B} T \sim 1 \mathrm{meV}\right.$ at $\left.T=10 \mathrm{~K}\right)$ to the chemical potentials of the $n$ - and $p$-doped contact layers, see Fig. 3(d).

For sample B1, our data also reveal an unusual blueshift of the broad EL emission band as the bias is decreased from $V=+1.6 \mathrm{~V}$ toward the flatband condition $(V \sim+1.5 \mathrm{~V})$, see arrow in the color plot of Fig. 3(c). This energy shift is consistent with a dominant recombination of electrons and holes from QD excited states. The presence of both ground and excited state QD emissions is also evident in the higher bias plots of Fig. 3(a).

From these results we conclude that in the low density samples, relaxation of carriers is less efficient compared to that in the high density case. Auger processes are known to play a fundamental role in the dynamics of carriers in QDs. ${ }^{15-17}$ Our present results suggest that this mechanism is enhanced in structures with a high density of small dots. In

these samples, carrier-carrier interaction in the dot/wetting layer and between neighboring dots is expected to be stronger because the electronic coupling between the dot and the wetting layer is facilitated by the spreading of the electronic wave function into the wetting layer and, in addition, the distance between nearest dots is small $(\sim 10 \mathrm{~nm})$. Auger processes can also lead to upconversion luminescence (UCL), i.e., emission from dots populated by carriers with energies higher than the excitation energy. UCL has been observed previously only in photoluminescence studies. ${ }^{18}$ Our observation of this effect in the EL of our high density dot structures will be discussed in detail elsewhere.

In conclusion, we have shown how voltage-tunable tunneling injection of carriers from the doped layers of $p-i-n$ diodes can be used to excite sharp-line EL from individual QDs within a large ensemble and to study the excitation and relaxation mechanisms of carriers in the dots. In the devices with low QD density, we have achieved resonant tunneling excitation of the dots with no significant energy relaxation of the electrically injected carriers, a technique that could be relevant for future spintronic and photonic applications.

This work is supported by the Engineering and Physical Sciences Research Council (United Kingdom) and the European Commission (SANDiE Network of Excellence, NMP4CT-2004-500101).

${ }^{1}$ Semiconductor Nanocrystals: From Basic Principles to Applications, edited by A. L. Efros, D. J. Lockwood, and L. Tsybeskov (Kluwer Academic/Plenum, New York, 2003).

${ }^{2}$ D. J. Mowbray and M. S. Skolnick, J. Phys. D 38, 2059 (2005)

${ }^{3}$ I. E. Itskevich, S. I. Rybchenko, I. I. Tartakovskii, S. T. Stoddart, A. Levin, P. C. Main, L. Eaves, M. Henini, and S. Parnell, Appl. Phys. Lett. 76, $3932(2000)$

${ }^{4}$ Z. Yuan, B. E. Kardynal, R. M. Stevenson, A. J. Shields, C. J. Lobo, K. Cooper, N. S. Beattie, D. A. Ritchie, and M. Pepper, Science 295, 102 (2002).

${ }^{5}$ X. L. Xu, D. A. Williams, and J. R. A. Cleaver, Appl. Phys. Lett. 85, 3238 (2004).

${ }^{6}$ V. Zwiller, T. Aichele, F. Hatami, W. T. Masselink, and O. Benson, Appl. Phys. Lett. 86, 091911 (2005).

${ }^{7}$ P. Michler, A. Kiraz, C. Becher, W. V. Schoenfeld, P. M. Petroff, L. D. Zhang, E. Hu, and A. Imamoglu, Science 290, 2282 (2000).

${ }^{8}$ N. Panev, A. I. Persson, N. Skold, and L. Samuelson, Appl. Phys. Lett. 83, 2238 (2003)

${ }^{9}$ M. T. Borgstrom, V. Zwiller, E. Muller, and A. Imamoglu, Nano Lett. 5, 1439 (2005).

${ }^{10}$ M. Kroutvar, Y. Ducommun, D. Heiss, M. Bichler, D. Schuh, G. Abstreiter, and J. J. Finley, Nature (London) 432, 81 (2004).

${ }^{11}$ S. Stufler, P. Ester, A. Zrenner, and M. Bichler, Phys. Rev. Lett. 96, 037402 (2006)

${ }^{12}$ K. Takehana, F. Pulizzi, A. Patanè, M. Henini, P. C. Main, L. Eaves, D. Granados, and J. M. Garcia, J. Cryst. Growth 251, 155 (2003).

${ }^{13}$ G. Kiesslich, A. Wacker, E. Scholl, S. A. Vitusevich, A. E. Belyaev, S. V. Danylyuk, A. Forster, N. Klein, and M. Henini, Phys. Rev. B 68, 125331 (2003).

${ }^{14}$ A. Patanè, A. Levin, A. Polimeni, L. Eaves, P. C. Main, M. Henini, and G. Hill, Phys. Rev. B 62, 11084 (2000).

${ }^{15}$ D. Morris, N. Perret, and S. Fafard, Appl. Phys. Lett. 75, 3593 (1999).

${ }^{16}$ R. Ferreira and G. Bastard, Appl. Phys. Lett. 74, 2818 (1999).

${ }^{17}$ F. Pulizzi, A. Kent, A. Patanè, L. Eaves, and M. Henini, Appl. Phys. Lett. 84, 3046 (2004).

${ }^{18}$ C. Kammerer, G. Cassabois, C. Voisin, C. Delalande, P. Roussignol, and J. M. Gerard, Phys. Rev. Lett. 87, 207401 (2001). 